\title{
Fußarbeit
}

HANDS-ON UND HANDS-OFF: MOBILISATION DER FÜSSE Fußschmerzen stehen mit

einer Lebenszeitprävalenzrate von 40 Prozent auf Platz 2 - gleich nach dem Rückenschmerz. Doch der therapeutische Methodenkoffer bei Fußbeschwerden ist oft karg bestückt. Einfache und wirkungsvolle Griffe und Übungen bietet die Spiraldynamik. Christian Larsen stellt zwei Hands-on-Techniken und zwei Übungen vor.

N ach wie vor unterteilen viele Orthopädie-Lehrbücher den Fuß in einen mobilen medialen und einen stabilen lateralen Teil (๑ Abb. 1). Messdaten aus kinematischen In-vivo-Untersuchungen der Eidgenössischen Technischen Hochschule Zürich belegen das Gegenteil [1, 2, 3]: Kuboid und Navikulare rotieren „quer“ um den Taluskopf und Kalkaneus. Somit verläuft die „Stabil-Mobil-Achse“ nicht längs zwischen 1.-3. Strahl und 4.-5. Strahl, sondern quer: Talus und Kalkaneus bilden den hinteren stabilen Teil, Navikulare und Kuboid stellen den mobilen Teil des Fußes dar (๑ Abb. 2).

Ebenso hartnäckig hält sich die Drei-Punkte-Theorie. Sie besagt, dass sich die Last im Fuß auf drei Punkten verteilt: Ferse, Großzehe und Kleinzehe. Auch das ist ein Irrtum. Richtig ist, dass der Vorfuß die Last flächig auf alle fünf Metatarsalköpfe und alle fünf Zehen verteilt. Hierfür ist eine aktive Exzentrik der gesamten intrinsischen Vorfußmuskulatur unter Belastung erforderlich.

Aufgrund dieser beiden Tatsachen ist es wichtig, bei Fußschmerzen folgende Komponenten zu überprüfen: Ist der Rückfuß stabil oder kommt es zum Einknicken nach innen (Hyperpronation in der frühen Standbeinphase) oder zum Wegkippen nach außen (Hypersupination in der späten Abrollphase)? Ist die artikuläre Beweglichkeit im Mittelfuß gewährleistet? Wie stark ist die Muskulatur im Vorfuß und wie beweglich ist das Quergewölbe? Je nachdem welche dieser Komponenten defizitär ist, entsteht ein Knick-, Hohloder Senk-/Plattfuß. Hierfür eignen sich die Fußspirale und die Fußwelle mit jeweiliger Eigenübung ( $\odot$ S. 32).

Christian Larsen

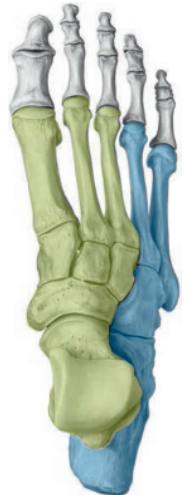

1

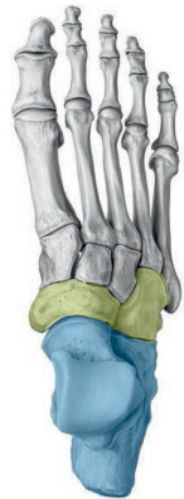

2
Abb. 1 Alte Unterteilung: Die "Stabil-Mobil-Achse“ läuft längs zwischen 1.-3. Strahl und 4.-5. Strahl. Der mediale Teil ist mobil (grün), der laterale Teil stabil (blau).

Abb. 2 Neue Unterteilung: Aktuelle Untersuchungen zeigen, dass Navikulare und Kuboid „quer" um Talus und Kalkaneus rotieren. Diese bilden somit den hinteren stabilen Teil, Navikulare und Kuboid den mobilen.
$\Rightarrow$ Das Literaturverzeichnis steht unter www.thieme-connect.de/ ejournals/physiopraxis > „Ausgabe 3/14“.

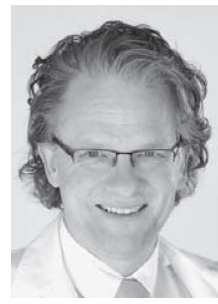

Dr. med. Christian Larsen studierte Medizin in Base und ist Leiter des Spiraldynamik Med Center Zürich. Er hat mehrere Bücher geschrieben, unter anderem den Patientenratgeber „Gut zu Fuß ein Leben lang“ und den Elternratgeber „Gesunde Füße für Ihr Kind“ erschienen im Trias-Verlag. Die dritte Auflage seines Buchs „Füße in guten Händen“ erscheint im Sommer 2014 im Thieme Verlag. Spiraldynamik ist eine registrierte Marke. 


\section{Hands-on: Fußspirale}

$>$ ASTE: Der Patient liegt in Rückenlage oder sitzt im Langsitz.

$>$ Griff: Der Therapeut umgreift mit einer Hand die Ferse des Patienten, mit der anderen Hand den Vorfuß (॰ Abb. 3). Das Sprunggelenk bleibt in Neutralnullstellung.

$>$ Mobilisation und Dosierung: Der Therapeut rotiert den Vor- und Rückfuß gegeneinander: den Vorfuß in Richtung Pronation, den Rückfuß im Sinne einer Supination. Anschließend löst er die „spiralige Verschraubung“ wieder. Verschraubung und Entschraubung wiederholt der Therapeut rhythmisch über drei bis fünf Minuten.

$>$ Wirkung: Die gegensinnige Verschraubung des Fußes um seine Längsachse gewährleistet die In-sich-Stabilität des Längsgewölbes: Der Rückfuß wird in orthograder Stellung stabilisiert (dies wirkt dem Knickfußmuster entgegen), die Fußwurzel- und Mittelfußknochen werden funktionell mobilisiert. Bei einem Senk-/Plattfuß wird das Längsgewölbe durch diese Technik verkürzt und die Fußsohlenmuskulatur tonisiert, bei einem Hohlfuß durch Traktion gestreckt und die Muskulatur detonisiert.

\section{Hands-off: Vier-Punkte-Stand}

$>$ ASTE: Der Patient steht im Zwei- oder Einbeinstand (๑ Abb. 4). Der Therapeut benötigt zwei Therabänder und legt je ein Ende eines Therabands lateral unter beide Fersen und medial unter beide Großzehengrundgelenke. Dann setzt er diese unter Zug, indem er die Bänder beispielsweise unter etwas einklemmt. Die Bänder dürfen nicht zu weit unter dem Fuß liegen, idealerweise nur wenige Millimeter.

$>$ Übung und Dosierung: Durch aktive Rückfuß-Supination (initiiert durch den M. tibialis posterior) und Vorfuß-Pronation (initiiert durch den M. peroneus longus) verstärkt der Patient den Fuß-Boden-Kontakt an den vier Druckpunkten und hält die Therabandenden fest. Dann führt der Therapeut Stör- und Ablenkungsmanöver durch wie Anschubsen, Bälle fangen oder Zahlenreihen aufsagen, bis der Patient einen oder mehrere Druckpunkte verliert. Die Übung kann zwischen einer und drei Minuten dauern - je nach gewünschtem Schwierigkeitsgrad. Besonders anspruchsvoll ist die Übung auf unebenem Boden.

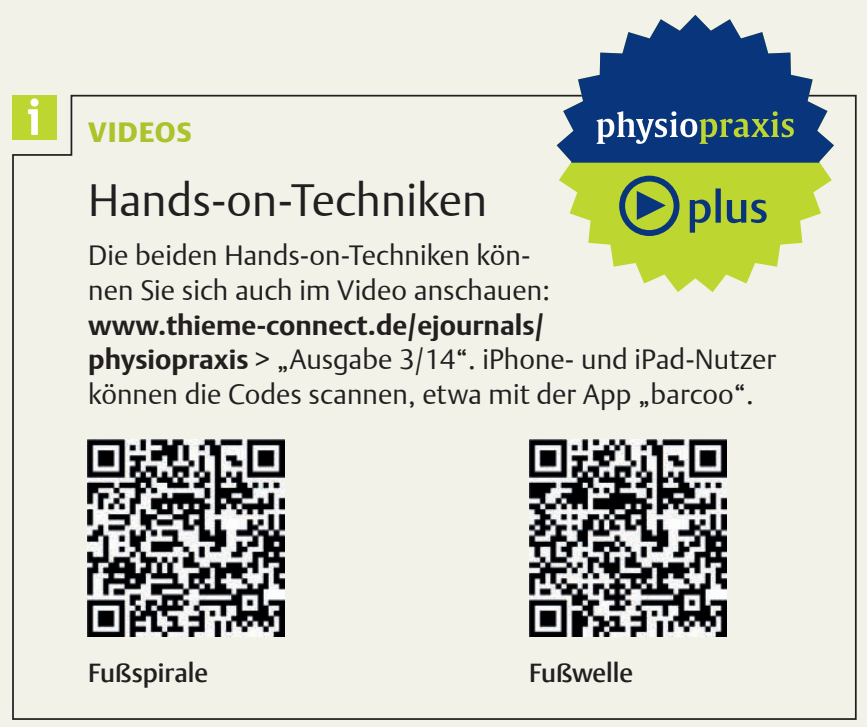

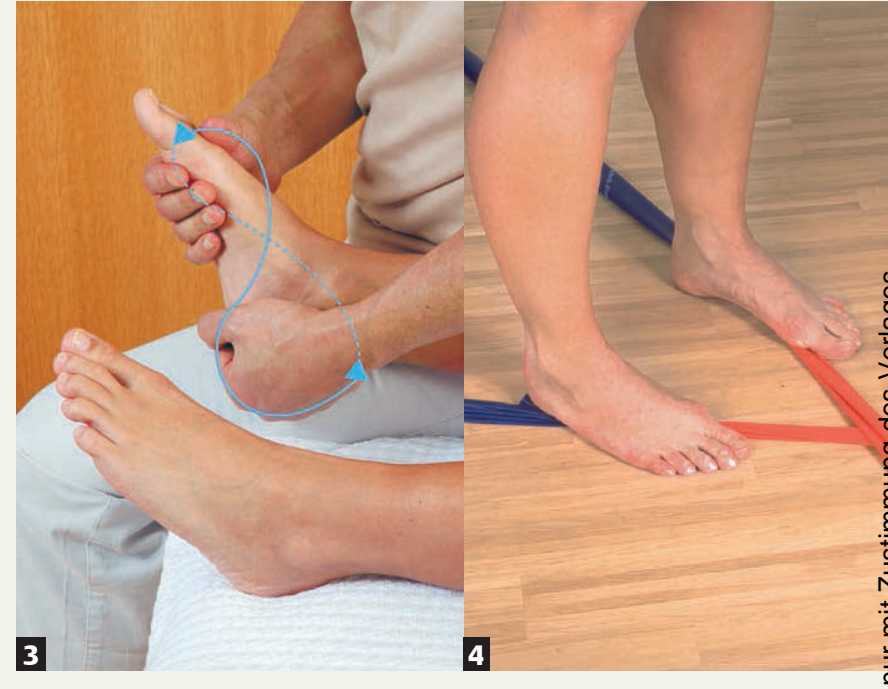

Abb. 3 Fußspirale: Der Therapeut bewegt den Rückfuß in Supination, den Vorfuß in Pronation. Im Anschluss eignet sich der Vier-Punkte-Stand.

Abb. 4 Vier-Punkte-Stand: Hierbei lernt der Patient, die durch die Mobilisation gewonnene rotatorische Beweglichkeit funktionell einzusetzen.

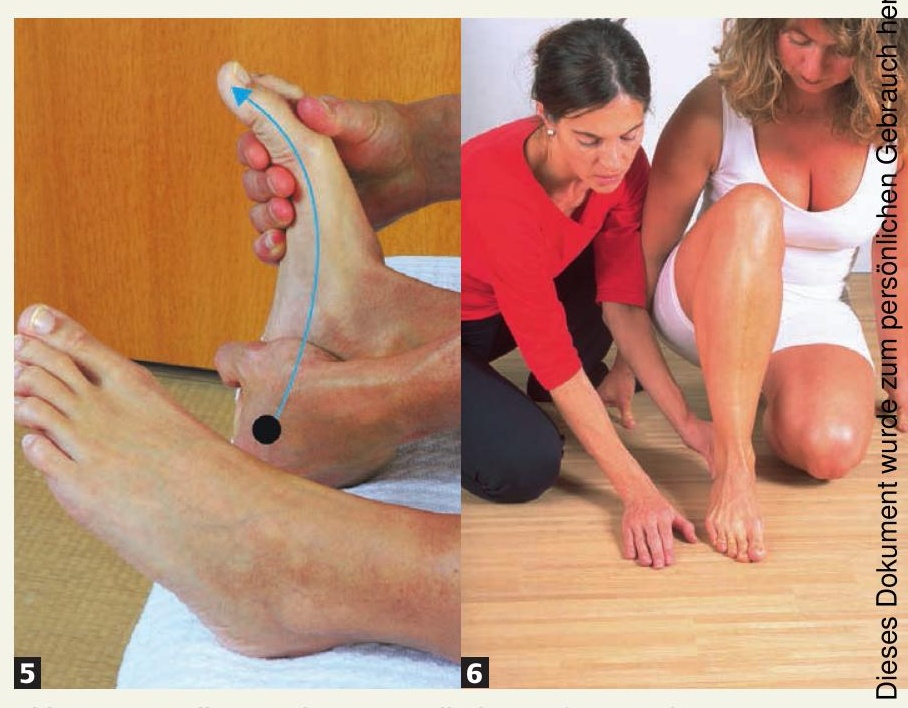

Abb. 5 Fußwelle: Der Therapeut rollt den Vorfuß in Richtung Ferse und wieder zurück. Danach eignet sich die Zehenraupe.

Abb. 6 Zehenraupe: Bei dieser Übung baut der Patient das muskuläre Vorfuß-Quergewölbe auf. Als Hilfe kann es ihm der Therapeut mit der Hand demonstrieren. So lernt der Patient, die durch die Fußwelle verbesserte Ansteuerung der Muskulatur funktionell zu nutzen. 


\section{Hands-on: Fußwelle}

$>$ ASTE: Der Patient liegt in Rückenlage oder sitzt im Langsitz.

$>$ Griff: Der Therapeut hält den Fuß wie bei der Fußschraube: Mit der einen Hand umgreift er die Ferse des Patienten, mit der anderen den Vorfuß (๑ Abb. 5). Das Sprunggelenk bleibt in Neutralnullstellung. Dann verschraubt er den Fuß spiralig um seine Längsachse (Vorfuß in Pronation, Rückfuß in Supination) und hält ihn dort.

$>$ Mobilisation und Dosierung: Der Vorfußgriff unterstützt das Quergewölbe (leichte Opposition von Groß- und Kleinzehe). Mit der Hand am Vorfuß vollzieht der Therapeut nun eine Wellenbewegung in Längsrichtung des Fußes: Das Einrollen erfolgt vom Vorfuß in Richtung Ferse, das Ausrollen in umgekehrter Richtung von hinten nach vorne. Diese Bewegung wiederholt der Therapeut rhythmisch über drei bis fünf Minuten.

> Wirkung: Das Einrollen des Fußes wirkt tonisierend, das Ausrollen detonisierend. Dadurch reguliert sich der Tonus der gesamten Fußsohlenmuskulatur. Beim Senk-/Plattfuß kann der Therapeut das tonisierende Einrollen betonen, dies stützt und stabilisiert das Längsgewölbe. Bei einem unbeweglichen Hohlfuß empfiehlt es sich, intensiv auszurollen, da dies detonisiert und mobilisiert.

\section{Hands-off: Zehenraupe}

$>$ ASTE: Der Patient stellt den zu beübenden Fuß auf und setzt sich auf die Ferse des anderen Beins (॰ Abb. 6).

$>$ Übung und Dosierung: Bei dieser Übung lernt der Patient das Quergewölbe am aufgestellten Fuß physiologisch einzustellen. Hat er einen Senk-/Spreizfuß, bewegt der Patient die Zehen wie fünf kleine Raupen in Richtung Ferse. So nehmen Quer- und Längsgewölbe zu, und der Fuß wird kürzer. Bei einem Hohl-/Spreizfuß ist es genau umgekehrt: Der Patient nimmt mit den Zehenspitzen Bodenkontakt auf und bewegt seine Zehen weg von der Ferse, die Ferse bleibt am Ort. Dadurch nehmen Quer- und Längsgewölbe ab, und der Fuß wird länger. Wichtig ist, dass der Patient nicht mit den Zehen krallt. Die Übung kann zwischen einer und drei Minuten dauern - je nach gewünschtem Schwierigkeitsgrad. Besonders anspruchsvoll ist die Übung auf unebenem Boden. Die intrinsische Ballenmuskulatur wird intensiv gekräftigt, wenn der Patient den Fuß teil- oder vollbelastet.

\section{Spiraldynamik} Körperarbeit

Wer mehr über Spiraldynamik erfahren möchte, kann mit etwas Glück das passende Buch „Spiraldynamik

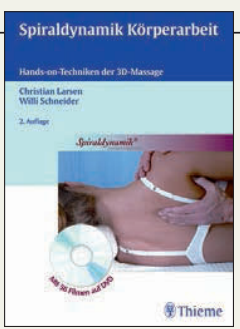
Körperarbeit“ aus dem Thieme Verlag gewinnen. Einfach unter www.thieme.de/physiopraxis > „Gewinnspiel“ auf das Stichwort „Spiraldynamik“ klicken. Der Einsendeschluss ist der 10.4.2014. Viel Glück. 